\title{
Perioperatives Management bei Patienten mit schlafbezogenen Atmungsstörungen
}

\section{Perioperative Management of Patients Suffering from Sleep-Related Breathing Disorders}

Autoren

Institute
F. Dette' ${ }^{1}$, M. Zoremba' ${ }^{1}$, J. Heitmann², S. Canisius ${ }^{2}$, H. Wulf ${ }^{1}$, U. Koehler ${ }^{2}$

Klinik für Anästhesie und Intensivtherapie (Direktor: Prof. Dr. H. Wulf)

2 Klinik für Innere Medizin, SP Pneumologie, Intensiv- und Schlafmedizin (Direktor: Prof. Dr. C. Vogelmeier), Philipps-Universität Marburg eingereicht 3.12.2008 akzeptiert nach Revision 25. 2. 2009

\section{Bibliografie}

DOI $10.1055 / \mathrm{s}-0029-1214508$

Online-Publikation: 15. 4. 2009

Pneumologie 2009; 63: 282-287

(c) Georg Thieme Verlag KG

Stuttgart · New York

ISSN 0934-8387

Korrespondenzadresse

Dr. med. Frank Dette

Klinik für Anästhesie und

Intensivtherapie

Universitätsklinikum Gießen

und Marburg GmbH

Standort Marburg

Baldingerstraße 1

35033 Marburg

Frank.Dette@web.de

\section{Zusammenfassung \\ $\nabla$}

Patienten mit schlafbezogenen Atmungsstörungen (SBAS) stellen innerhalb des operativen Gesamtkollektivs eine besondere Risikogruppe dar und sind während der perioperativen Phase in zwei von einander unabhängigen Perioden vital gefährdet. Während sich in der postoperativen Frühphase noch Überhänge von Narkosesubstanzen negativ auf die Atemarbeit auswirken, wird die postoperative Spätphase besonders durch Veränderungen des REM-Schlafanteils in der Nacht und damit assoziierten Herz-Kreislaufdysregulationen charakterisiert. Um ein sicheres perioperatives Management von Patienten mit SBAS gewährleisten zu können, müssen lückenloses Monitoring $\left(\mathrm{O}_{2}\right.$-Sättigung und $\left.\mathrm{pCO}_{2}\right)$ sowie die Möglichkeit der postoperativen nicht-invasiven Beatmung garantiert werden.

\section{Einleitung}

Allgemeinanästhesie und operative Eingriffe führen zu einer Beeinträchtigung der Atemmechanik mit einer Verschlechterung des pulmonalen Gasaustausches. Das Spektrum reicht von einer temporären Desaturation bis hin zu einer nachhaltigen Hypoxie und kann sowohl direkt als auch indirekt den postoperativen Verlauf der Patienten beeinflussen. Während die Verschlusskapazität (closure capacity) durch Allgemeinanästhesie weitgehend unbeeinflusst bleibt, kommt es unter maschineller Beatmung in Rückenlage zu einer Abnahme der Funktionellen Residualkapazität (FRC) von bis zu $20 \%$. Die Folge ist der Verschluss kleiner Atemwege mit Ausbildung von Atelektasen [1]. Diese sind bei über $90 \%$ der erwachsenen Patienten bereits kurz nach Narkoseinduktion im CT nachweisbar und können mehrere Tage persistieren.

\section{Abstract \\ $\nabla$}

Sleep-related breathing disorders have been associated with increased perioperative morbidity and mortality. The respective patients are at risk during two independent periods. Besides an early period, characterised by the influence of anaesthetics, patients are at risk also during a late period, which is characterised by nocturnal desaturation and disturbances of the cardiovascular system, caused by interference with the sleep architecture, especially of the REM sleep. To assure a safe perioperative management, a close monitoring $\left(\mathrm{O}_{2}\right.$ saturation and $\left.\mathrm{pCO}_{2}\right)$ and the option for non-invasive ventilation have to be guaranteed. sitas, obstruktive Schlafapnoe, Adipositas-Hypoventilations-Syndrom, COPD Stadien III/IV nach GOLD (resp. Globalinsuffizienz), Herzinsuffizienz

Ziel dieser Übersichtsarbeit ist es, auf die Problematik von schlafbezogenen Atmungsstörungen in der perioperativen Phase einzugehen und Konsequenzen für die operative und anästhesiologische Versorgung der Patienten aufzuzeigen.

Folgende Faktoren sind hinsichtlich peri- oder postoperativ auftretender respiratorischer Komplikationen von besonderer Relevanz:

- pharmakologische Effekte von Anästhetika, Sedativa und Analgetika

- Ausprägung und Länge der chirurgischen Intervention

- Morbidität (Begleiterkrankungen) wie Adipo- 


\section{Schlafbezogene Atmungsstörungen (SBAS)}

SBAS beschreiben eine Gruppe pathologischer Veränderungen der Atmung im Schlaf. Wichtige Vertreter sind die Schlafapnoe und das Adipositas-Hypoventilations-Syndrom. SBAS sind verbunden mit erhöhter Morbidität und Mortalität und stellen einen unabhängigen Risikofaktor für arteriellen Bluthochdruck [2,3] sowie kardio- [4-6] und zerebrovaskuläre [7-9] Erkrankungen dar. Schlafapnoe kann je nach zugrunde liegender Ursache in eine zentrale Atemregulationsstörung (zentrale Schlafapnoe, CSA), eine intermittierende Obstruktion der oberen Atemwege (obstruktive Schlafapnoe, OSA) und in Mischformen unterteilt werden.

\section{Obstruktive Schlafapnoe (OSA)}

Mit einer Prävalenz von etwa 5\% $[10,11](\mathrm{AHI} \geq 15)$ ist die obstruktive Schlafapnoe (OSA) die häufigste schlafbezogene Atmungsstörung [12]. Bislang existieren keine Daten zur Prävalenz von Schlafapnoe in einem operativen Gesamtkollektiv [13]. Pathophysiologisch liegt der obstruktiven Schlafapnoe ein rezidivierend auftretender Tonusverlust der oropharyngealen Muskulatur zugrunde, welcher eine Atemflusslimitation bis hin zum kompletten Verschluss der oberen Atemwege bei erhaltenem zentralen Atemstimulus zur Folge hat. Durch zentrale Weckreaktionen (Arousals) kommt es zu einer Wiedereröffnung der oberen Atemwege durch einen Anstieg des Muskeltonus, was zu einer Störung der Makro- und Mikrostruktur des Schlafes sowie einem erhöhten Sympathikotonus führt. Die an die Atmungsstörung gekoppelten Mechanismen wie intrathorakale Druckschwankungen, Veränderungen der Blutgase (Hypoxie und Hyperkapnie), Arousalreaktionen sowie die neurohumoral vermittelten Adaptationsvorgänge sind als kausal für die kardiovaskulären Folgeschäden anzusehen.

\section{Zentrale Schlafapnoe (CSA)}

Bei der zentralen Schlafapnoe können prinzipiell zwei Formen unterschieden werden. Zum einen die eigentliche zentrale Schlafapnoe (CSA), die durch immer wiederkehrende Atemstillstände in Folge einer Abnahme des zentralen Atemantriebs gekennzeichnet ist (kein Atemfluss, keine Atembewegungen). Diese Atmungsstörungen treten häufig im Übergang zwischen Wachzustand und Einschlafen (Leichtschlaf) auf. Zum anderen gibt es die Sonderform der Cheyne-Stoke-Atmung (CSR), die durch eine periodische Atmung (sog. Crescendo-Decrescendo-Muster) gekennzeichnet ist und gehäuft bei Patienten mit Herzinsuffizienz gefunden wird. CSR ist charakterisiert durch einen Wechsel aus Phasen mit Hypoventilation (bis hin zur Apnoe) und darauf folgenden Phasen der Hyperventilation. Pathophysiologisch basiert die Abfolge von Hypo- und Hyperventilationsphasen bei der CSR wahrscheinlich auf einem ständig wiederkehrenden Unterschreiten der Apnoeschwelle durch eine Hypokapnie mit konsekutiver Hyperventilationsphase. Vermutlich bedingt durch eine verlängerte Kreislaufzeit bei Herzinsuffizienz werden die Änderungen der Blutgase verzögert registriert, weshalb es immer wieder zu einem Wechsel zwischen Hypokapnie (mit der Folge der Hypoventilation und Apnoe) und Hyperkapnie (mit der Folge einer Hyperventilation) kommt [14]. Neben einer Herzinsuffizienz können auch Opioide eine zentrale Schlafapnoe auslösen. Hier stehen dann die zentralnervös wirkenden atemdepressiven Effekte der Opioide im Vordergrund. Zusätzlich führen Opioide aber auch zu einer Störung der Schlafstruktur wie zum Beispiel zu einer Unterdrückung des REM-Schlafs. Die durch die zentralen
Atmungsstörungen verursachten Weckreaktionen führen darüber hinaus zur vermehrten Übergängen zwischen Wachzustand und Schlaf, so dass dann wiederum gehäuft zentrale Atmungsstörungen auftreten können.

\section{Obesitas-Hypoventilations-Syndrom (OHS)}

Die Definition des OHS umfasst Obesitas (BMI $\left.>30 \mathrm{~kg} / \mathrm{m}^{2}\right)$, Hyperkapnie (oftmals bedingt durch eine alveoläre Hypoventilation) im Wachzustand $\left(\mathrm{pCO}_{2}>45 \mathrm{~mm} \mathrm{Hg}\right.$ bei Ausschluss anderweitiger Ursachen der Hypoventilation) und hauptsächlich obstruktiven Atmungsstörungen im Schlaf. Bei Patienten mit OHS kommt es im Schlaf sowohl zum Auftreten von OSA als auch von vorwiegend REM-Schlaf assoziierten Hypoventilationen.

\section{Risikostratifizierung für respiratorische Komplikationen}

Patienten mit schlafbezogenen Atmungsstörungen sind perioperativ in zwei von einander unabhängigen Phasen vital gefährdet: Die erste Phase beginnt direkt postoperativ und erstreckt sich vom Ende der Operation bzw. der Extubation bis zum Wiedererlangen der vollständigen Vigilanz. Eine zweite Phase erstreckt sich über die zweite Hälfte der ersten postoperativen Woche.

\section{A - Postoperative Frühphase}

Neben patientenbezogenen Risikofaktoren wie Rauchen, Adipositas, Lungenerkrankungen, Herzinsuffizienz oder Schlafapnoe spielen in der postoperativen Frühphase zudem verfahrensassoziierte Risikofaktoren eine besondere Rolle. Nach Smetana et al. [15] prädisponieren mit einer Odds Ratio (gepooled) von 6,90 $(2,74-17,36)$ bzw. 4,24 (2,89-6,23) vor allem operative Eingriffe an Aorta und Thorax zum Auftreten von respiratorischen Komplikationen, gefolgt von abdominal-, neuro- und gefäßchirurgischen Eingriffen. Weitere Risikofaktoren stellen Notfalleingriffe (Odds Ratio 2,52 [1,69-3,75]), lange OP-Zeit über mehr als 3 $4 \mathrm{~h}$ (Odds Ratio 2,26 [1,47-3,47]), Allgemeinanästhesie (Odds Ratio 2,35 [1,77-3,12]) und Transfusionen (Odds Ratio 1,47 $[1,26-1,71])$ dar $[15,16]$.

\section{Der Einfluss der Allgemeinanästhesie in der postoperativen} Frühphase

Während der Frühphase üben noch bestehende Effekte verabreichter Substanzen wie Opioide, Sedativa und Muskelrelaxanzien einen negativen Einfluss auf die Atemregulation und Atemarbeit des Patienten aus [17]. Insbesondere Substanzen wie Benzodiazepine [18] und Disoprivan (Propofol) [19,20] führen zu einer Abschwächung des muskulären Tonus im Oropharynx. Somit sind nicht nur Patienten mit bekannter Schlafapnoe der Gefahr einer postoperativen Atemwegsverlegung ausgesetzt. Ähnliche Probleme, wenn auch in deutlich reduziertem Ausmaß, sind nach Verwendung von volatilen Anästhetika wie Isoflurane, Sevoflurane oder Desflurane beschrieben [21]. Entscheidend für eine suffiziente Atemwegskontrolle in dieser Phase ist ein schnelles Wiedererlangen der vollständigen Vigilanz des Patienten. Volatile Anästhetika, insbesondere Desflurane, scheinen hier im Vergleich zu Propofol einen deutlichen Vorteil zu bieten [22]. 
Der Einfluss von Schmerz und Schmerztherapie in der postoperativen Frühphase

Schmerz führt zu Schonatmung mit reduzierten Tidalvolumina und einer Reduktion des Hustenstoßes. Konsekutiv kommt es zu vermehrter Atelektasenbildung und Sekretansammlung im Bronchialsystem. Opioide stellen einen wichtigen Bestandteil der postoperativen Schmerztherapie dar, beeinflussen über eine Reduktion des zentralen Atemantriebs die Lungenfunktion in dieser Phase jedoch negativ. Somit sollte, wenn möglich, die Schmerztherapie z.B. durch eine Epiduralanästhesie (EDA) mit Lokalanästhetika ergänzt werden.

\section{B - Postoperative Spätphase}

Die postoperative Spätphase erstreckt sich über die Nächte der zweiten postoperativen Wochenhälfte und ist in erster Linie gekennzeichnet durch Veränderungen der Schlafarchitektur mit erhöhten Anteilen des REM-Schlafs (Rebound Phänomen) sowie durch nächtliche Hypoxieperioden.

Pulmonale, kardiovaskuläre und zerebrale Komplikationen treten postoperativ gehäuft während dieser Zeit und überwiegend nachts auf [23 - 25]. Auch wird in diesem Zeitraum eine besondere Häufung von Delir-Zuständen („Durchgangssyndrom“) und postoperativen kognitiven Defiziten beschrieben $[26,27]$. Der Häufigkeitsgipfel für Myokardinfarkte liegt zwischen dem zweiten und fünften postoperativen Tag $[28,29]$. Sowohl die Narkosemedikation als auch das Trauma des operativen Eingriffs nehmen Einfluss auf den Schlaf, infolge dessen Apnoephasen mit Desaturationen auch bei schlafgesunden Patienten beobachtet werden können. Während Komplikationen der postoperativen Frühphase überwiegend auf den Einfluss der Narkosemedikation zurückgeführt werden können, sind für Komplikationen der Spätphase besonders Dysregulationen des REM-Schlafmusters verantwortlich.

\section{REM-Schlaf und postoperativer REM-Rebound}

Beim erwachsenen Menschen nimmt der REM-Schlaf etwa $20-$ $25 \%$ des Nachtschlafs ein. Der relative Anteil der REM-Phasen am gesamten Schlafzyklus nimmt in den frühen Morgenstunden zu. Die drei REM-charakteristischen Kriterien sind schnelle Augenbewegungen, gemischte EEG-Frequenzen sowie ein erniedrigter Muskeltonus. Als phasisches REM werden die Anteile des REM-Schlafs mit einer hohen Dichte an raschen Augenbewegungen bezeichnet, während diese Augenbewegungen in Phasen mit tonischem REM nicht auftreten, jedoch weiterhin die REM-Kriterien zutreffen. Der Tonus der quergestreiften Muskulatur ist mit Ausnahme von Herz, Zwerchfell und Augen deutlich herabgesetzt und niedriger als in allen anderen Schlafstadien [11]. Der REMSchlaf ist durch eine erhöhte Variabilität der Herz-Kreislauf- und Atmungsparameter gekennzeichnet. Die Chemosensitivität und die Atemantwort auf hypoxische und hyperkapnische Reize sind reduziert $[11,48]$.

Körperlicher und psychischer Stress, ungewohnte Umgebung, peri- und postoperativer Schmerz sowie noch persistierende medikamentöse Anästhetikaeffekte können die Makro- und Mikrostruktur des Schlafes nachhaltig stören. So kommt es postoperativ zu einer reduzierten Schlafeffizienz mit erniedrigten Anteilen an Tief- und REM-Schlaf. Mitunter werden die REM-Perioden gänzlich unterdrückt. Untersuchungen des Schlaf-EEG's zeigen eine Suppression der nahezu gesamten REM-Aktivität während der ersten und teilweise auch während der zweiten und dritten postoperativen Nacht $[11,30]$. Im weiteren Verlauf kommt es zum verstärkten Auftreten von REM-Schlaf, dem sogenannten
REM-rebound. REM-rebound bezeichnet eine Zunahme an Menge, Dauer und Dichte von REM-Schlaf. Dieses Phänomen erscheint, wenn REM-Schlaf unterdrückende Effekte (z.B. Operation, Stress, Schmerzen) entfallen [31]. So findet sich ein REMRebound typischerweise auch nach dem Absetzen von trizyklischen Antidepressiva oder MAO-Hemmern [32] und unter einer erfolgreich eingeleiteten nichtinvasiven Beatmungstherapie bei Patienten mit obstruktiver Schlafapnoe. Vor dem Hintergrund, dass REM-Schlaf zu einer verminderten Kontrolle der autonomen Funktionen führt, ist die Wahrscheinlichkeit des Auftretens von respiratorischen und kardiovaskulären Ereignissen (Hypoxämien, Herzrhythmusstörungen, myokardiale und cerebrale Ischämien, Blutdruckschwankungen) erwartungsgemäß erhöht. Der speziell im REM-Schlaf auftretende Tonusverlust der quergestreiften Muskulatur im Oropharynx kann zur Zunahme der Häufigkeit von Apnoe- und Hypopnoephasen führen.

Die Ursachen des postoperativen REM-Rebound sind bislang nicht hinreichend geklärt. Neben dem Einfluss der genannten Narkose-Substanzen werden körpereigene, durch das Operationstrauma bedingte Stressreaktionen diskutiert [30]. Inwiefern die Faktoren Narkose, Operationstrauma oder die Kombination zur Suppression des postoperativen REM-Schlafs mit REM-Rebound in den Folgenächten beitragen, konnte bislang nicht hinreichend geklärt werden.

\section{Konsequenzen für das perioperative Management $\nabla$}

Bei Patienten mit SBAS ist postoperativ mit einer erhöhten Rate an pulmonalen und kardiovaskulären Komplikationen zu rechnen [23-25]. Eine wichtige Voraussetzung für ein adäquates peri- und postoperatives Management ist demzufolge primär die Identifikation von Risikopatienten [11,34-36].

In $\bullet$ Tab. 1 findet sich eine Zusammenfassung der Empfehlungen für das perioperative Management in Anlehnung an Empfehlungen der American Society of Anesthesiologists (ASA).

\section{A - Präoperativ}

Zur Risikoeinstufung ist eine Anamnese unter besonderer Berücksichtigung schlafbezogener Atmungsstörungen sinnvoll und notwendig. Im Rahmen der Prämedikationsvisite sollte nach Symptomen wie lautem Schnarchen, plötzlichem Erwachen mit Erstickungsgefühl, Tagesschläfrigkeit und fremdanamnestisch bekannten nächtlichen Atempausen gefragt werden. Die klinische Untersuchung sollte Befunde wie einen kurzen, dicken Hals, nasale Obstruktionen, Tonsillenhypertrophie, Retrognathie sowie einen engen Oropharynx berücksichtigen [29,37-41]. Von einigen Autoren werden Fragebögen als Screeningmedium favorisiert [39, 42,43].

Bei anamnestischem Verdacht auf das Vorliegen einer SBAS sollte wegen der Gefahr einer präoperativen oropharyngealen Atemwegsverlegung beim nicht überwachten Patienten auf eine Prämedikation mit Benzodiazepinen verzichtet werden.

Patienten mit OSA sowie begleitender arterieller Hypertonie, koronarer Herzerkrankung, Herzinsuffizienz und Zustand nach Apoplex sind als Risikopatienten innerhalb des Gesamtkollektivs einzustufen. Ist zudem noch eine Lungenerkrankung vorbekannt oder aufgrund der klinischen Diagnostik wahrscheinlich, sollte präoperativ eine Lungenfunktion zur Bestimmung der pulmonalen Reservekapazitäten mit Blutgasanalyse initiiert werden. Anhand der arteriellen Blutgasanalyse kann eine Beurteilung des Schweregrades der Gasaustauschstörung vorgenommen und das 
Tab. 1 Kurzgefasst - Empfehlungen für das perioperative Management [49].

\section{Präoperativ}

Fragen nach vorbekannter SBAS und BeatmungsTherapie (z. B. cPAP)

Fragen nach Schlafverhalten:

(1) Aufwachen mit Erstickungsgefühl

(2) Fremdanamnestisch Atempausen und

lautes Schnarchen

(3) Tagesmüdigkeit

Körperliche Merkmale wie:

(1) Dicker, kurzer Hals

(2) Retrognathie

(3) Tonsillenhypertrophie

Weiterführende Diagnostik wie Poly(somno)-

graphie und Blutgasanalyse bei dringendem

Verdacht auf OSA/AHS etc.

CPAP-Therapie schon präoperativ einleiten bzw.

fortführen, insbesondere bei schwerer Schlafapnoe

\section{Intraoperativ}

Vorbereitung auf einen potenziell schwierigen Atemweg - Fiberoptik bereit halten.

Intubation und Extubation mit erhöhtem Oberkörper

Geeignetes Anästhesieverfahren wählen.

Wenn möglich, Regionalverfahren bevorzugen

Gute Atemwegssicherung garantieren

Kapnographie, insbesondere beim sedierten

Patienten

Extubation nur beim wachen Patienten ohne nachweisbaren Relaxanzüberhang

\section{Postoperativ}

Lückenloses Monitoring der Atem- und Kreislaufparameter; nach Möglichkeit auch $\mathrm{pCO}_{2}$ !

Zurückhaltender Einsatz von Opioiden in der postoperativen Schmerztherapie

Sauerstoffgabe, bis $\mathrm{SaO}_{2}$-Baseline unter Raumluft gehalten werden kann, cave: $\mathrm{CO}_{2}$ !

CPAP-Therapie zur Sicherstellung einer suffizienten Ventilation

Patientenlagerung mit erhöhtem Oberkörper

Atmungs-Monitoring auch während der folgenden Nächte
Vorliegen einer respiratorischen Insuffizienz belegt werden. Kann trotz präoperativer medikamentöser und physiotherapeutischer Maßnahmen keine Optimierung der respiratorischen Situation erzielt werden, so sollte eine postoperative Beatmungsmöglichkeit sichergestellt werden (invasiv oder non-invasiv). Eine präoperative nicht-invasive Beatmung (NIV) kann in bestimmten Situationen eine sinnvolle Ergänzung zur präoperativen Optimierung von Risikopatienten darstellen, zur Rechtfertigung einer generellen Empfehlung zur NIV in der präoperativen Vorbereitung existieren keine ausreichenden klinischen Daten [1].

\section{B - Intraoperativ}

Eine Patientenlagerung mit erhöhtem Oberkörper wirkt sich günstig auf die Narkoseeinleitung aus [44], da diese die Kollapsneigung des Oropharynx reduziert und gleichzeitig den Druck des Abdomens auf das Zwerchfell vermindert. Dies führt konsekutiv zu einer Reduktion des inspiratorischen Beatmungsdrucks und somit zu einer leichteren Maskenbeatmung. Bei Patienten mit Schlafapnoe muss aufgrund der anatomischen Begebenheiten mit einem schwierigen Atemweg gerechnet werden [4547]. Dies kann sich zum einen durch eine erschwerte Maskenbeatmung, aber auch durch schlechte Sichtverhältnisse bei der endotrachealen Intubation manifestieren, so dass vor Narkoseeinleitung alternative Hilfsmittel zur Atemwegssicherung bereitgestellt werden sollten. Hierbei soll auch auf die Leitlinie zum Airway Management der Deutschen Gesellschaft für Anästhesiologie und Intensivmedizin (DGAI) verwiesen werden [48].

Besonders bei Wahl eines Regionalanästhesieverfahrens hat die intraoperative Überwachung (Kapnographie) und Sicherung der Atemwege oberste Priorität [49]. Eine intraoperativ auftretende Atemwegsobstruktion mit konsekutiver Apnoe konnte auch unter ausschließlicher Spinalanästhesie beobachtet werden. Vor diesem Hintergrund sollte bei Patienten eine supportive Sedierung in Verbindung mit einer Regionalanästhesie kritisch hinterfragt werden. Auch wenn die Datenlage bezüglich der Wahl des „idealen Narkoseverfahrens“ bei Patienten mit SBAS noch nicht eindeutig ist, scheinen diese bei peripheren Eingriffen von Regionalverfahren zu profitieren [49].

Ist ein Allgemeinanästhesieverfahren gewählt worden, ist es essenziell, dass der Patient erst nach Wiedererlangen der vollständigen Atemwegskontrolle sowie der normalen Muskelaktivität (nach Relaxation) extubiert wird. Zur Beurteilung des richtigen
Extubationszeitpunktes stehen eine zielgerichtete motorische Reaktion, Kopfheben nach Aufforderung, Relaxometrie und adäquates Tidalvolumen unter Spontanatmung (ggf. mit Unterstützung durch CPAP/ASB) als hilfreiche Kriterien zur Verfügung. $\mathrm{Zu}$ bedenken ist allerdings, dass es trotz acceleromyographischem neuromuskulärem Monitoring zu nicht unerheblichen Restrelaxierungen kommen kann. Auch während der Extubation wirkt sich aus genannten Gründen eine Patientenlagerung mit erhöhtem Oberkörper günstig aus.

\section{C - Postoperativ}

Bei der Ausrichtung des postoperativen Managements müssen postoperative Früh- und Spätphase getrennt voneinander betrachtet werden.

\section{C.1 - Postoperative Frühphase}

In der Frühphase stehen Schmerztherapie, Oxygenierung, Lagerung und Monitoring, dessen Bestandteil eine $\mathrm{pCO}_{2}$-Messung sein sollte, im Vordergrund.

\section{Monitoring}

Neben den üblichen Parametern wie Atemfrequenz, Herzfrequenz- und -rhythmus, Blutdruck und Sauerstoffsättigung (siehe auch Leitlinie „postoperative Überwachung“ der DGAI) stellt die Messung des $\mathrm{pCO}_{2}$ bei Patienten mit schlafbezogenen Atmungsstörungen einen sinnvollen Parameter dar. Nur über die Messung des $\mathrm{pCO}_{2}$ kann die alveoläre Ventilation adäquat beurteilt werden [51 -53]. Unter Sauerstoffapplikation kann eine Hypoventilation durch eine vermeintlich gute Sauerstoffsättigung maskiert werden.

\section{Sicherstellung einer suffizienten Schmerztherapie}

Um eine schmerzbedingte Schonatmung mit niedrigen Tidalvolumina und reduzierter Hustenkapazität zu vermeiden, ist eine suffiziente Schmerztherapie in der postoperativen Frühphase ein essentieller Bestandteil des Managements.

Im Rahmen der Schmerztherapie sollte der Einsatz von Opioiden aufgrund des bekannten depressiven Effekts auf den Atemantrieb kritisch geprüft werden. Verfahren ohne Opioidzusatz sollte der Vorzug gegeben werden. Besonders bei operativen Eingriffen an Thorax und Abdomen hat sich die Epiduralanästhesie (EDA) als supportives Verfahren zur suffizienten intra- und postoperativen Schmerztherapie bewährt. In mehreren Metaanalysen konnten 
unter EDA niedrigere Raten pulmonaler Komplikationen (z.B. Pneumonie) und niedrigere Schmerzlevel bei gleichzeitig geringerem Opioidbedarf gezeigt werden [54-56].

Veränderungen des postoperativen REM-Schlafmusters mit REM-Rebound und nächtlicher Hypoxie werden jedoch auch unter EDA (mit Opioidzusatz) nicht verhindert. Lundstrom et al. [55] konnten an 50 Patienten nach Coronar-Bypass-Operation in der postoperativen Spätphase, am Ende der ersten postoperativen Woche, keinen signifikanten Unterschied zwischen Patienten nach Allgemeinanästhesie mit und ohne EDA zeigen. Es kam in beiden Gruppen, sowohl mit PDK als auch ohne, zu nächtlicher Hypoxie. Lässt man andere positive Effekte der EDA außer acht, manifestiert sich der Vorteil der EDA besonders in der postoperativen Frühphase.

\section{Sicherstellung einer suffizienten Oxygenierung}

Eine schnelle Wiederherstellung der Lungenfunktion hinsichtlich der Lungenvolumina ist ein entscheidender Faktor zur Minimierung von postoperativer respiratorischer Insuffizienz. Da nur bei Aufrechterhaltung einer suffizienten Ventilation eine adäquate Oxygenierung gewährleistet werden kann, sollte in dieser Phase ein nicht-invasives Beatmungskonzept Berücksichtigung finden. Nach einer Empfehlung (E27) einer aktuellen S3-Leitlinie zur non-invasiven Beatmung (NIV) können bei Patienten mit erhöhtem Risiko für eine postoperative respiratorische Insuffizienz durch frühzeitige Anwendung von CPAP (Continuous Positive Airway Pressure) bzw. NIV unmittelbar nach der Extubation die Reintubationsrate und weitere Komplikationen signifikant gesenkt werden [1]. Bei Patienten mit vorbekannter SBAS und bereits eingeleiteter Heimbeatmung sollte das eigene CPAP/BIPAPGerät eingesetzt werden $[49,44]$. Arisaka et al. [57] konnten zeigen, dass bei OSA-Patienten durch frühzeitigen, kontinuierlichen Einsatz von nasaler CPAP-Therapie (nCPAP) die Atemwege offen gehalten werden, und somit ein sicherer Einsatz von Allgemeinanästhesie und opioidbasierter Schmerztherapie in diesem Patientenkollektiv gewährleistet werden kann.

\section{Zentrale Atemregulationsstörungen}

Es existieren bislang keine ausreichenden klinischen Daten, die das postoperative Management von Patienten mit zentralen Atemregulationsstörungen betreffen. In mehreren Arbeiten konnte jedoch gezeigt werden, dass im nicht operativen Kontext auch durch kurzfristigen Einsatz von NIV in Form von BiPAP (Bilevel Positive Airway Pressure Support) die Anzahl der zentralen Apnoephasen mit konsekutiver Sauerstoffentsättigung signifikant gesenkt werden kann. Ein reines CPAP-Verfahren ist effektiv gegen obstruktive Atmungsstörungen, nicht aber kurzfristig gegen zentrale $[58,59]$. Dies gilt sowohl für Herzinsuffizienz-, als auch für Opioid-induzierte zentrale Atmungsstörungen [60]. Ein neues Therapieverfahren für Cheyne-Stokes-Atmung stellt die Adaptive Servoventilation (ASV) dar. Wenn ein Patient keine Atemarbeit mehr zeigt, generiert das Atemtherapiegerät, das grundsätzlich in einer Auto-CPAP-Funktion arbeitet, einen kurzen, steilen Impuls, der auf das bestehende Druckniveau addiert wird. Somit wird die zentrale Atempause unterbrochen.

\section{C.2 - Postoperative Spätphase}

Das Management der postoperativen Spätphase ist von Empfehlungen in der Literatur bislang nahezu unberücksichtigt. Empfehlungen der ASA beziehen sich praktisch ausschließlich auf die Frühphase [49]. Für die Spätphase wird eine Überwachung mittels Pulsoximetrie empfohlen. Über die zeitliche Dauer finden sich keine einheitlichen Angaben. Auf den Seiten der DGAI ist dieses Thema bislang gänzlich unbeachtet. Im Hinblick auf erwartete Dysregulationen des Herz-Kreislaufsystems gegen Mitte bis Ende der ersten postoperativen Woche erscheint bei Patienten mit bekannter oder gemutmaßter OSA eine Fortführung einer nächtlichen NIV-Therapie sinnvoll. Bislang existieren diesbezüglich jedoch keine ausreichenden klinischen Daten.

\section{Fazit}

$\nabla$

Patienten mit schlafbezogenen Atmungsstörungen sind mit einer hohen Dunkelziffer bislang nicht diagnostizierter Erkrankungen als besondere Risikogruppe innerhalb eines operativen Patientenkollektivs einzustufen und bedürfen besonderer Beachtung. Erweiterte Hilfsmittel zur Sicherung schwieriger Atemwegsverhältnisse und ein lückenloses Monitoring sowie die Möglichkeit einer postoperativen NIV sind essentielle Bestandteile eines perioperativen Managements dieser Patienten.

\section{Literatur}

1 Schönhofer B, Kuhlen R, Neumann P et al. Nichtinvasive Beatmung als Therapie der akuten respiratorischen Insuffizienz. Pneumologie 2008; 62: $449-479$

2 Lavie P, Silverberg D, Oksenberg A, Hoffstein V. Obstructive sleep apnea and hypertension: from correlative to causative relationship. J Clin Hypertens (Greenwich) 2001; 3: 296-301

3 Lavie P, Herer P, Hoffstein V. Obstructive sleep apnoea syndrome as a risk factor for hypertension: population study. BMJ 2000; 320: 479 482

4 Bradley TD, Floras JS. Sleep apnea and heart failure: Part II: central sleep apnea. Circulation 2003; 107: $1822-1826$

5 Bradley TD, Floras JS. Sleep apnea and heart failure: Part I: obstructive sleep apnea. Circulation 2003; 107: 1671-1678

6 Caples SM, Garcia-Touchard A, Somers VK. Sleep-disordered breathing and cardiovascular risk. Sleep 2007; 30: 291-303

7 Arzt M, Young T, Finn L et al. Association of sleep-disordered breathing and the occurrence of stroke. Am J Respir Crit Care Med 2005; 172: $1447-1451$

8 Culebras A. Sleep apnea and stroke. Rev Neurol Dis 2005; 2: $13-19$

9 Wierzbicka A, Rola R, Wichniak A et al. The incidence of sleep apnea in patients with stroke or transient ischemic attack. J Physiol Pharmacol 2006; 57: 385 - 390

10 Bixler EO, Vgontzas AN, Lin HM et al. Prevalence of sleep-disordered breathing in women: effects of gender. Am J Respir Crit Care Med 2001; 63: 608-613

11 Kaw R, Michota F, Jaffer A et al. Unrecognized sleep apnea in the surgical patient: implications for the perioperative setting. Chest 2006; 129: $198-205$

12 Parati G, Lombardi C, Narkiewicz K. Sleep apnea: epidemiology, pathophysiology, and relation to cardiovascular risk. Am J Physiol Regul Integr Comp Physiol 2007; 293: R1671 - R1683

13 Chung SA, Yuan H, Chung F. A systemic review of obstructive sleep apnea and its implications for anesthesiologists. Anesth Analg 2008; 107: $1543-1563$

14 Yumino D, Bradley TD. Central sleep apnea and Cheyne-Stokes respiration. Proc Am Thorac Soc 2008; 5: 226-236

15 Smetana GW, Lawrence VA, Cornell JE. American College of Physicians. Preoperative pulmonary risk stratification for noncardiothoracic surgery: systematic review for the American College of Physicians. Ann Intern Med 2006; 144: 581-595

16 Qaseem A, Snow V, Fitterman N et al. Clinical Efficacy Assessment Subcommittee of the American College of Physicians. Risk assessment for and strategies to reduce perioperative pulmonary complications for patients undergoing noncardiothoracic surgery: a guideline from the American College of Physicians. Ann Intern Med 2006; 144: 575-580

17 Mogri M, Khan MI, Grant BJ, Mador MJ. Central sleep apnea induced by acute ingestion of opioids. Chest 2008; 133: 1484-1488 
18 Bailey PL, Pace NL, Ashburn MA et al. Frequent hypoxemia and apnea after sedation with midazolam and fentanyl. Anesthesiology 1990; 73: $826-830$

19 Eastwood PR, Platt PR, Shepherd K et al. Collapsibility of the upper airway at different concentrations of propofol anesthesia. Anesthesiology 2005; 103: $470-477$

20 Eikermann M, Malhotra A, Fassbender P et al. Differential effects of isoflurane and propofol on upper airway dilator muscle activity and breathing. Anesthesiology 2008; 108: 897 - 906

21 Moote CA, Knill RL. Isoflurane anesthesia causes a transient alteration in nocturnal sleep. Anesthesiology 1988; 69: 327 - 331

22 Juvin P, Vadam C, Malek L et al. Postoperative recovery after desflurane, propofol, or isoflurane anesthesia among morbidly obese patients: a prospective, randomized study. Anesth Analg 2000; 91: 714-719

23 Gupta RM, Parvizi J, Hanssen AD et al. Postoperative complications in patients with obstructive sleep apnea syndrome undergoing hip or knee replacement: a case-control study. Mayo Clin Proc 2001; 76: $897-905$

24 Hwang D, Shakir N, Limann B et al. Association of sleep-disordered breathing with postoperative complications. Chest 2008; 133: 1128 1134

25 Reeder MK, Muir AD, Foëx P et al. Postoperative myocardial ischaemia: temporal association with nocturnal hypoxaemia. Br J Anaesth 199; 67: $626-631$

26 Aakerlund LP, Rosenberg J. Postoperative delirium: treatment with supplementary oxygen. Br J Anaesth 1994; 72: 286-290

27 Rosenberg J, Oturai P, Erichsen CJ et al. Effect of general anesthesia and major versus minor surgery on late postoperative episodic and constant hypoxemia. J Clin Anesth 1994; 6: 212-216

28 Steen PA, Tinker JH, Tarhan S. Myocardial reinfarction after anesthesia and surgery. JAMA 1978; 239: 2566 - 2570

29 Stradling JR, Crosby JH. Predictors and prevalence of obstructive sleep apnoea and snoring in 1001 middle aged men. Thorax 1991; 46: 85 90

30 Rosenberg-Adamsen S, Kehlet H et al. Postoperative sleep disturbances: mechanisms and clinical implications. Br J Anaesth 1996; 76: 552 - 559

31 Knill RL, Moote CA, Skinner MI et al. Anesthesia with abdominal surgery leads to intense REM sleep during the first postoperative week. Anesthesiology 1990; 73: $52-61$

32 Cohen RM, Pickar D, Garnett D et al. REM sleep suppression induced by selective monoamine oxidase inhibitors. Psychopharmacology 1982; 78: $137-140$

33 Kavey NB, Ahshuler KZ. Sleep in herniorrhaphy patients. Am J Surg 1979; 138: 683 - 687

34 Hallowell PT, Stellato TA, Schuster M et al. Potentially life-threatening sleep apnea is unrecognized without aggressive evaluation. Am J Surg 2007; 193: 364 - 367

35 Sharma SK, Kurian S, Malik Vet al. A stepped approach for prediction of obstructive sleep apnea in overtly asymptomatic obese subjects: a hospital based study. Sleep Med 2004; 5: 351 - 357

36 Shaw IR, Lavigne G, Mayer P et al. Acute intravenous administration of morphine perturbs sleep architecture in healthy pain-free young adults: a preliminary study. Sleep 2005; 28: 677-682

37 Blake DW, Chia PH, Donnan G et al. Preoperative assessment for obstructive sleep apnoea and the prediction of postoperative respiratory obstruction and hypoxaemia. Anaesth Intensive Care 2008; 36: 379 384

38 Davies RJ, Ali NJ, Stradling JR. Neck circumference and other clinical features in the diagnosis of the obstructive sleep apnoea syndrome. Thorax 1992; 47: $101-105$

39 Gali B, Whalen FXJr, Gay PC et al. Management plan to reduce risks in perioperative care of patients with presumed obstructive sleep apnea syndrome. J Clin Sleep Med 2007; 3: 582-588
40 Hoffstein V, Szalai JP. Predictive value of clinical features in diagnosing obstructive sleep apnea. Sleep 1993; 16: 118-122

41 Hoffstein V, Mateika S. Differences in abdominal and neck circumferences in patients with and without obstructive sleep apnoea. Eur Respir J 1992; 5: 377 - 381

42 Sharma SK, Vasudev C, Sinha S et al. Validation of the modified Berlin questionnaire to identify patients at risk for the obstructive sleep apnoea syndrome. Indian J Med Res 2006; 124: 281 - 290

43 Netzer NC, Stoohs RA, Netzer CM et al. Using the Berlin Questionnaire to identify patients at risk for the sleep apnea syndrome. Ann Intern Med 1999; 131: 485 - 491

44 Kaw R, Aboussouan L, Auckley D et al. Challenges in pulmonary risk assessment and perioperative management in bariatric surgery patients. Obes Surg 2008; 18: 134-138

45 Benumof JL. Obesity, sleep apnea, the airway and anesthesia. Curr Opin Anaesthesiol 2004; 17: $21-30$

46 Benumof JL. Obstructive sleep apnea in the adult obese patient: implications for airway management. Anesthesiol Clin North America 2002 20: $789-811$

47 Hiremath AS, Hillman DR, James AL et al. Relationship between difficult tracheal intubation and obstructive sleep apnoea. Br J Anaesth 1998; 80: $606-611$

48 Deutsche Gesellschaft für Anästhesiologie und Intensivmedizin. Leitlinie Airway Management. Anästh Intensivmed 2004; 45: 302 - 306

49 Gross JB, Bachenberg KL, Benumof JL et al. American Society of Anesthesiologists Task Force on Perioperative Management. Practice guidelines for the perioperative management of patients with obstructive sleep apnea: a report by the American Society of Anesthesiologists Task Force on Perioperative Management of patients with obstructive sleep apnea. Anesthesiology 2006; 104: 1081-1093

50 Deutsche Gesellschaft für Anästhesiologie und Intensivmedizin. Leitlinie zur postoperativen Überwachung. Anästh Intensivmed 1989; 39 $202-203$

51 Janssens JP, Laszlo A, Uldry C et al. Non-invasive (transcutaneous) monitoring of PCO2 (TcPCO2) in older adults. Gerontology 2005; 51: 174 178

52 Janssens JP, Howarth-Frey C, Chevrolet JC et al. Transcutaneous PCO2 to monitor noninvasive mechanical ventilation in adults: assessment of a new transcutaneous PCO2 device. Chest 1998; 113: 768-773

53 Senn O, Clarenbach CF, Kaplan V et al. Monitoring carbon dioxide tension and arterial oxygen saturation by a single earlobe sensor in patients with critical illness or sleep apnea. Chest 2005; 128: 1291 - 1296

54 Liu SS, Block BM, Wu CL. Effects of perioperative central neuraxial analgesia on outcome after coronary artery bypass surgery: a meta-analysis. Anesthesiology 2004; 101: $153-161$

55 Lundstrøm LH, Nygård E, Hviid LB et al. The effect of thoracic epidural analgesia on the occurrence of late postoperative hypoxemia in patients undergoing elective coronary bypass surgery: a randomized controlled trial. Chest 2005; 128: $1564-1570$

56 Lawrence VA, Cornell JE, Smetana GW; American College of Physicians. Strategies to reduce postoperative pulmonary complications after noncardiothoracic surgery: systematic review for the American College of Physicians. Ann Intern Med 2006; 144: 596-608

57 Arisaka H, Sakuraba S, Kobayashi R et al. Perioperative management of obstructive sleep apnea with nasal continuous positive airway pressure. Anesth Prog 2008; 55: 121 - 123

58 Arzt M, Wensel R, Montalvan S et al. Effects of dynamic bilevel positive airway pressure support on central sleepapnea in men with heart failure. Chest 2008; 134: $61-66$

59 Naughton MT, Lorenzi-Filho G. Sleep in heart failure. Prog Cardiovasc Dis 2009; $51: 339-349$

60 Alattar MA, Scharf SM. Opioid-associated central sleep apnea: a case series. Sleep Breath 2008; Sep 20 [Epub ahead of print] 